\title{
Spatial positioning of individuals in a group of feral horses: a case study using drone technology
}

\section{$\operatorname{AUTHOR}(\mathrm{S})$ :}

Inoue, Sota; Yamamoto, Shinya; Ringhofer, Monamie; Mendonça, Renata S.; Pereira, Carlos; Hirata, Satoshi

\section{CITATION:}

Inoue, Sota ... [et al]. Spatial positioning of individuals in a group of feral horses: a case study using drone technology. Mammal Research 2018, 64(2): 249-259

\section{ISSUE DATE:}

2018-04

URL:

http://hdl.handle.net/2433/240619

\section{RIGHT:}

This is a post-peer-review, pre-copyedit version of an article published in Mammal Research. The final authenticated version is available online at: http://dx.doi.org/10.1007/s13364-018-0400-2:; The full-text file will be made open to the public on 6 October 2019 in accordance with publisher's 'Terms and Conditions for Self-Archiving.; この論文は出版社版 でありません。引用の際には出版社版をご確認ご利用ください。; This is not the published version. Please cite only the published version. 
Spatial positioning of individuals in a group of feral horses: a case study using drone technology

3 Sota Inoue $^{\mathrm{a}^{*}}$, Shinya Yamamoto ${ }^{\mathrm{b}}$, Monamie Ringhofer ${ }^{\mathrm{b}}$, Renata S. Mendonça ${ }^{\mathrm{c}}$, Carlos Pereira $^{\mathrm{d}} \&$ Satoshi

4 Hirata $^{\mathrm{a}}$

5

6

${ }^{a}$ Wildlife Research Center, Kyoto University, 2-24 Tanaka Sekiden-cho, Sakyo, Kyoto 606-3201, Japan.

${ }^{b}$ Kyoto University Institute for Advanced Study, Yoshida Ushinomiya-cho, Sakyo, Kyoto 606-8501, Japan.

'Primates Research Institute, Kyoto University, 41-2 Kanrin, Inuyama 484-8506, Japan.

${ }^{d}$ Université Paris III Sorbonne Nouvelle, 13 rue Santeuil, 75005, Paris, France

\section{*Correspondence:}

Mr. Sota Inoue

Wildlife Research Center, Kyoto University

2-24 Tanaka Sekiden-cho, Sakyo, Kyoto 606-8203, Japan

E-mail: sota.inoe@gmail.com; Tel: +81-75-771-4398

\section{Acknowledgements}

Special thanks are due to Viana do Castelo city for supporting our project. We are also grateful to Agostinho Costinha, the director of Descubra Minho, Lourenço Almada of Associação O Caminho do Garrano. We also thank the villagers in Montaria for their support during our stay, Tetsuro Matsuzawa for the generous guidance throughout the study, and Dora Biro and Valéria Romano for helpful comments on an earlier version of our manuscript. The study was financially supported by grants from the Japan Society for the Promotion of Science (JSPS core-to-core CCSN and JSPS-LGP-U04 to Tetsuro Matsuzawa, KAKENHI Nos. 15H01619 and 15H05309 to Shinya Yamamoto) and the Ministry of Education, Culture, Sports, Science, and Technology in Japan (MEXT No.16H06283 to Tetsuro Matsuzawa). We thank Lilly Gray and Adam Phillips, PhD from Edanz Group (www.edanzediting.com/ac) for editing a draft of this manuscript. 
30 Keywords: Drone; Horse; Nearest neighbor; Repulsion and Attraction; Spatial position; Social 31 relationship 


\section{Introduction}

From ants to elephants, some organisms form groups and others live largely in solitude. Social living may improve foraging efficiency if individuals share information about food availability and location.

Furthermore, forming a group has other benefits that depend on the needs of each species. For example, it might serve to reduce predation risk, reduce energy consumption related to air or water resistance, or improve reproduction efficiency (Barak and Yom-Tov 1989; Herskin and Steffensen 1998; Weimerskirch et al. 2001; Cameron et al. 2009).

One theory predicts that individuals at the periphery of a group are at higher risk of predation than individuals in central positions (Hamilton 1971). Some researchers have argued that food gains, as well as predation risk, are often higher at the peripheries. Thus, animals must at some level consider the tradeoff between predation risk and foraging benefits when choosing their spatial position within a group (Morrell and Romey 2008). As a result, risk of predation and degree of feeding competition, for example, can vary with respect to spatial position (Hirsch 2007).

Previous studies investigating spatial positioning in animals have focused on groups of numerous species, including schools of fish, flocks of birds, and groups of mammals (Rhine et al. 1985; van Schaik and van Noordwijk 1987; Janson 1990; Hirsch 2011; Ryder et al. 2012; Teichroeb et al. 2015). Important social dynamics within animal groups that potentially affect individual spatial position often include dominance, friendships, and kin relationships. For example, movement of individual social mammals is strongly influenced by their social relationships such that the dominant or older individuals are more likely to become the leader in the leader-follower relationship (King et al. 2011; Sueur and Deneubourg 2011; Andrieu et al. 2016; Tokuyama and Furuichi 2017). In addition to social relationships, physical relationships between individuals within a space (i.e., distance, proximity, and orientation) need to be taken into account when considering animal movement because they might be a mediating factor between the relationships and movements. Researchers have investigated proximity among conspecifics within groups and found that shorter inter-individual distance indicates closer relationships in Japanese macaques (Furuichi 1983) and that proximity correlated with the reproductive states of female rhesus monkeys (Czaja et al. 1975). Similar aspects have been observed in horses. Crowell-Davis et al. (1986) showed that the sex difference between foals and their nearest neighbors changes as the foals develop 
60 (Crowell-Davis et al. 1986). Kimura (1988) found that the partners who frequently accompany the nearest

61 neighbor were not the same as those who participate in mutual grooming among females (Kimura 1998).

62 Using spatial-positioning data, Bourjade et al. (2015) and Briard et al. (2015) reported that there might be no leaders on collective departure in domestic horses and the influence of a stallion on the group behavior is not strong (Bourjade et al. 2015; Briard et al. 2015).

To obtain data on spatial positions, researchers recently have started using bio-logging technologies in which GPS devises are mounted on animals or video recordings are taken from relatively high above ground and are analyzed using image processing (Lukeman et al. 2010a; Rosenthal et al. 2015; Farine et al. 2017). These studies have shown rules with respect to the distance and angle between individuals within a group, suggesting strong preference and anisotropy of the direction for neighboring individuals (Ballerini et al. 2008a; Lukeman et al. 2010a; Katz et al. 2011; Pettit et al. 2013; Rosenthal et al. 2015). Researchers have further suggested that the spatial area surrounding a single individual in a group can be divided into three zones: attraction (two individuals move toward each other when they are too far apart), orientation (two individuals tend to face the same direction), and repulsion (two individuals avoid each other when they are too close) (Couzin et al. 2002).

In the case of large mammals in the wild however, attaching GPS devices to all group members or recording video that covers the locations of all group members from high enough above the ground is difficult (e.g., if video recordings are made from human eye height, recording orientation and the masking of individuals by other animal makes high accuracy analysis of individual spatial positions difficult). Although studies have investigated spatial relationships in mammals including horses, they have been primarily conducted with captive animals that were confined to a relatively small area or they relied on visual judgements of the distance between individuals, which are often categorical (e.g., less than $1 \mathrm{~m}$ or greater than $5 \mathrm{~m}$ ) (Krueger et al. 2014; Briard et al. 2015). Recently, researchers succeeded in obtaining location data from wild baboons by mounting GPS devices on them (Strandburg-Peshkin et al. 2015; Farine et al. 2017), but the data were not collected from all group members, which exemplifies the difficulty of attaching GPS devices to wild mammals. To obtain data from GPS devices, animals must first be captured and they must weigh enough to carry the device. Thus, the question remains: are there 
attraction-repulsion areas or anisotropy of nearest neighbors) for spatial positioning of individual fish within schools or birds within flocks also applicable to wild mammals? Quantitative data on spatial positioning is imperative for understanding the processes and rules that generate variation in the patterns exhibited across and within taxa. To address this question, we conducted the present case study using drones - unmanned aerial vehicles that are remotely controlled by an operator - to focus on large and feral mammals. Although researchers have already used drones to examine some species of wild and domestic animals (Vermeulen et al. 2013;Chrétien et al. 2016; Goolsby et al. 2016; Torney et al. 2016; Jung 2017), most of these studies focused on detecting and monitoring them from the perspective of conservation and management, and few have focused on animal behavior (Ozogány and Vicsek 2014).

Using drones enables the recording of all members of a group from the sky, which is optimal for analyzing spatial positions, as long as no obstacles come between the drone and subject animals. Feral horses meet the first requirement of this methodology because they usually live in relatively monotonous plains that are covered with grasses and herbs, as opposed to complex three-dimensional spaces that include tall trees or thick bushes that can block a drone's line of sight. Horses usually form long-term stable harems comprising multiple members (Berger 1977; Klimov 1988), which are comparable to some primate species that also form stable groups with fixed members (Nishida 1968; Harcourt 1978; Kano 1982; Linklater and Cameron 2009).

In the present study, we investigated several characteristics related to spatial position in a group of feral horses as a case study for clarifying the nature of spatial positions within a mammal group. We investigated characteristics that have been commonly investigated in the case of fish schools and bird flocks, including the relative positions of each individual in the group, inter-individual distances, and the distances and angles to the nearest neighbors (Lukeman et al. 2010a; Strandburg-Peshkin et al. 2013; Rosenthal et al. 2015). Additionally, we tested for correlation between social networks measured by grooming frequency (which has previously been used as an indicator of friendship association) and spatial position, as a study by Kimura (1998) suggested a possibility that these two might differ. Our focus was on analyzing stationary spatial positions because fast and relatively long-distance movement of individual feral horses makes positional analysis difficult for a number of reasons (e.g., short battery life and the drone's own movement). We focused on stationary spatial positions during foraging, a situation in which 
horses do not engage in fast or long-distance movement. The present study had two primary aims: (1) to describe the basic characteristics of stationary spatial positions within a group of horses and (2) to clarify how social networks in a group influence spatial position.

\section{Material and Methods}

\subsection{Study site and animals}

The study was conducted in June 2016 at Serra D’Arga, an 825 m high mountain located in northern Portugal $\left(8^{\circ} 42^{\prime} \mathrm{N}, 41^{\circ} 48^{\prime} \mathrm{E}\right.$ ), which was established as a new research site in 2016 (see Ringhofer et al. 2017 for details) (Figure 1). This region has a Mediterranean climate, and the horse habitat includes a grass field, rocky ground, a forest, and shrub areas. More than 200 feral Garrano horses live in this region (Ringhofer et al. 2017). The focal group comprised one adult male (Uzumasa), seven adult females (Uji, Katsura, Gion, Fushimi, Kishiwada, Ayabe, and Akashi), and two foals born in the spring of 2016. One adult female (Akashi) was in the process of transferring from another group; this female moved back and forth between the two groups throughout the observation period. All members of the group were identifiable by their appearance. Analysis was conducted during the period when this individual was present in the group.

The target group had two foals. The orientation and distance of foals depended on those of their mothers. We excluded data from these two foals as our goal was to analyze the positions and relationships of independent adult horses. A separate analysis including the foals is provided as Supplementary Material.

The field observations complied with the guidelines for animal studies in the wild issued by the Wildlife Research Center of Kyoto University, Japan.

\subsection{Data collection}

Photos of the target group were taken every 30 min using an unmanned aerial vehicle (drone, Phantom3 Advanced, DJI, China) (Figure 2). The vehicle was a quadcopter with a video camera (1080p resolution) that was operated remotely with the camera angle set perpendicular to the ground. The drone took off approximately $10-50 \mathrm{~m}$ from the horses and flew at an altitude of $25-80 \mathrm{~m}$. Advance test flights 
confirmed that as long as the drone stayed at least $10 \mathrm{~m}$ away from the horses, they did not run away, panic, or show any other behavioral responses to the drone. Because horses did not move rapidly, position data from two consecutive scans would not be independent if the scan interval was too short. Therefore, we set the scan interval at 30 min to avoid this potential for correlated horse positions.

While photos were taken, the positions of each individual were recorded from the ground by observers who could identify individuals. When horses were located in the shadows of trees, we stopped taking photos, and began again after $30 \mathrm{~min}$. Three to thirteen images were acquired per day, and a total of 102 images were acquired during our 13-day observation. Observation lasted $4-10 \mathrm{~h}$ per day, for a total of $88.5 \mathrm{~h}$ over the course of the study.

\subsection{Behavioral data collection}

We also recorded aggressive behavior and grooming events using all-occurrence sampling (Altmann 1974). These recordings took place regardless of whether or not the drone was flown. Grooming and aggressive behaviors performed against or by foals were not analyzed. Similar to a previous study with horses (Heitor et al. 2006), aggressive behaviors included biting, chasing, kicking, and striking were considered indicators of social rank within the group. We then calculated the frequency of aggressive events per hour and per individual.

\subsection{Image categorization}

We excluded images from further analysis if they did not contain all individuals. Additionally, individuals from other groups were occasionally present with the target group, and such cases were also excluded from analysis. Group activities were divided into three categories: (1) travel (the entire group walked/ran toward a certain direction, often in a line), (2) rest (more than half the group rested motionlessly, usually packed in a small space), and (3) forage (group members foraged freely). We determined the category of activity based on observations before and after taking the drone photos, and then excluded instances of travel and rest from the analysis. This resulted in 61 foraging images $(60 \%$ of the total number of images) for further analysis. 


\subsection{Correction of camera distortion}

The distortion of the camera lens was corrected using the lens filter function in Photoshop CC (Adobe Systems incorporated) selecting the FC300 camera property for DJI Phantom 3, which was provided by Photoshop CC as a preset value. We confirmed the accuracy of the correction by taking a photo of a checkerboard grid with a known absolute size and applying the lens-distortion correction. We then calculated the error by comparing the length of the grid in the photo with its actual length. Before we corrected the distortion using Photoshop CC, the maximum error was 12\% and around the periphery of the photo. After correction, it was 3\% or less. This was acceptable, and we thus based our analysis on the distortion-corrected images.

\subsection{Calculation of distance and direction}

We used three measures to characterize the spatial positions of individuals within the group: interindividual distance, nearest neighbor distance, and the distance between each individual and the center of the group. This latter value was calculated based on the x-y pixel coordinates of each individual's location in a $3200 \times 4000$ pixels photo, with the top left of an image being the origin $(0,0)$. The group center was defined as the average of all individual coordinates. We defined an individual's location as the midpoint between the tip of the head and the base of the tail. Average body length (BL) of all individuals was used as the unit of length for further analysis in measuring the distance between individuals. This was because the drone's ground height varied, and the scale of the images was thus different in each photograph (i.e., the length of a pixel represented a different absolute length in the real environment) because no standard object of known size could be placed in the photos. The assumption here is that because all members were present in all photos that we analyzed, the average body length of all individuals should remain constant across photos. Body length was the distance between the base of the tail and the base of the neck. The reason for not using the tip of the head was that head orientation varied depending on the posture of the horses. When horses were grazing in the field, they lowered their head. In contrast, the distance from the base of the neck to the base of the tail was relatively constant and always straight, as viewed from above via the drone. 
vectors from the base of the tail to the tip of the head. Although several previous studies have adopted one body length $(\leq 1.5 \mathrm{~m})$ as the category that best estimates the strength of the interaction between two horses, we defined "proximity" as two individuals being within 2 BL of each other. This was because our method measures the distance between the center point of one individual to that of another, not the edge of one individual to the edge of another, as was the case in previous studies (Waring 1983; Heitor and Oom 2006). Our study remains comparable to these previous studies because 2 BLs using our method is effectively equivalent to $1 \mathrm{BL}$ in the Waring (1983) and Heitor and Oom (2006) studies (the distance from the central point of the body and its outer surface is roughly $0.5 \mathrm{BL}$, which is then multiplied by two individuals), with the proviso that the two measures are not actually identical because the animals are not circular in shape. Next, to investigate how the orientation of an individual to its nearest neighbor depends on position (front and rear, viewed from the target individual), we calculated the inner product between the unit vector of an individual (from the tail base to the head) and that of its nearest neighbor. Higher inner product values indicate greater orientation alignment.

\subsection{Random test}

Under complete spatial randomness, individual positions in local areas follow a Poisson distribution and the average distance to the nearest neighbor follows a Weibull distribution (Fortin et al. 2002). To measure the randomness of individual positions, we used the following index:

$$
q=\frac{d \sqrt{n}}{\pi r^{2}}
$$

where $r$ is the average distance to the nearest neighbor for all individuals in an image, $n$ is the number of individuals, and $d$ represents the distance between the group center and the furthest individuals. If $q$ equals 0.5 , horse positions are random, if it is close to 0 , positions are in a limited smaller area, and if it is close to 1, positions have some non-random regularity (Skellam 1952; Pollard 1971). 
To estimate the strength of association between two individuals, we used measures of grooming and proximity frequency. Mutual grooming and proximity are generally used to estimate the positive relationship between individuals (Briard et al. 2015). We calculated simple ratio indices (SRI) for each measure that were defined as follows:

$$
S R I=\frac{x}{x+y_{a}+y_{b}}
$$

where $x$ represents the frequency of grooming or the proximity between individuals $a$ and $b, y_{a}$ is the frequency of grooming or the proximity between individual $a$ and other individuals, and $y_{b}$ is the same as $y_{a}$, but for individual $b$ (Cairns and Schwager 1987). Additionally, we calculated a distance index (DI) from the average distance between each pair of individuals:

$$
D I=\frac{-D_{a b}+0.5\left(D_{a}+D_{b}\right)}{0.5\left(D_{a}+D_{b}\right)}
$$

where $D_{a b}$ represents the average distance between individuals $a$ and $b$, and $D_{a}$ and $D_{b}$ represent the maximum distance between individuals $a$ and $b$ and other individuals, respectively. In this way, we obtained three weighted (from 0 to 1) indices of interactions (grooming, proximity, and distance). We then measured eigenvector centrality to clarify the influence of each individual in these networks with Ucinet 6.0 software (Borgatti et al. 2002).

\subsection{Statistical analysis}

Statistical tests were performed with R 3.3.2 (R Development Core Team 2016). With respect to the distribution of the distance between individuals, we first determined whether the distribution followed a Weibull distribution based on the reasoning described above. However, visual inspection of the data indicated a gamma distribution; thus, we tested whether it followed a gamma distribution. With respect to the social network, we used Ucinet 6.0 to conduct Quadratic Assignment Procedure (QAP) tests to assess the influence of grooming on proximity and distance. We used 5000 permutations in the QAP tests.

\section{Results}

3.1 Aggressive behavior 
At this research site, the frequency of aggressive behaviors was very low. We observed only 10 aggressive behaviors over the course of the study, and average frequency per individual per hour was 0.03. This was significantly lower (Kruskal-Wallis test, $\chi^{2}=28.718, d f=3, p<0.01$ ) than what was reported in previous studies of feral horses at other sites (Heitor and Vicente 2008; Keiper 1988; Weeks et al. 2000; the frequencies of aggressive behaviors were 2.43, 5.9, and 0.4, respectively). Therefore, social rank could not be determined statistically.

\subsection{Relative locations of individuals}

Analyzing the distance of each individual from the central point revealed that the mean distance was significantly greater for the male (Uzu) than for five of the seven females (Figure 3; Steel test, $n=61$, Uzu-Uji: $t=5.48, p<0.01$, -Kis: $t=4.19, p<0.05$, -Kat: $t=4.26, p<0.05$, -Aya: $t=1.89, p=0.95,-$ Gio: $t=4.44, p<0.05$, -Fus: $t=2.65, p=0.61$, -Aka: $t=4.65, p<0.01$ ). We did not find any significant differences in female positioning regarding the center vs. the periphery (ANOVA, $n=61, d f=6, F=2.03$, $p=0.06)$.

\subsection{Distribution of distance between individuals}

The distribution of inter-individual distances could indicate whether horse positions are random or related to aggregation or diffusion. The histogram of all inter-individual distances followed a gamma distribution (shape $=2.24$, rate $=0.172 ;$-S test, $n=1708, D=0.06, p=0.11$ ) (Fig. 4), while that for nearest-neighbor distances did not follow a gamma distribution (shape $=1.83$, rate $=0.31 ; \mathrm{K}-\mathrm{S}$ test, $n=488, D=0.1394, p$ $<0.01$ ) or a Weibull distribution (shape $=1.18$, scale $=6.38$; K-S test, $n=488, D=0.14, p<0.01$ ) (Fig.

4). The peak of the nearest-neighbor histogram was shifted left compared with that of the all-pair histogram. We excluded the male from q-value calculation because he was located in the periphery. Figure 5 shows the q-value histogram. The peak was around 0.5 , but the cases less than 0.5 outnumbered those greater than 0.5 .

\subsection{Positioning and orientation of the nearest neighbor}


Rather than being uniform, the density plot for the nearest-neighbor data was biased depending on the angle (K-S test, $n=444, D=1, p<0.01$ ). Figure 6 shows a density plot of nearest neighbors within $5 \mathrm{BL}$ that is magnified from a density plot within $10 \mathrm{BL}$. The plot shows that the nearest neighbor tended to be located toward the sides of a horse more frequently than toward the back or front (Fig. 6).

Next, the ratio of inner products larger than 0 to those less than 0 was smaller when the nearest neighbor was located behind a horse than when it was located in front (prop test, $n=132, \chi^{2}=$ $1.56, d f=1, p=0.10)$. Thus, when the nearest neighbor was behind a horse, the target individual was less frequently orientated in a similar direction. Further, the minimum and average values for the rear (mean: 0.62, minimum: -0.64 ) were higher than those for the front (mean: 0.44, minimum: -0.99 ), whereas the maximum values were the same (both were 1).

\subsection{Social network analysis}

Three social networks were drawn from the three quantified social interactions: grooming, proximity $(<2$ BL; see Methods), and inter-individual distance (Fig. 7). The proximity and inter-individual distance networks trended towards a correlation, although it was not statistically significant (QAP test, $r=0.30, p$ $=0.09)$. This result was expected because removing auto-correlation between the two networks was not possible. We found a significant correlation between the grooming and inter-individual distance networks (QAP test, $r=0.51, p<0.01$ ), but not between the grooming and proximity networks (QAP test, $r=$ $-0.14, p=0.27)$.

For each network, we next calculated the eigenvector centrality, which indicates power in a network. Individuals with high eigenvector centralities were different in each of the three networks. Regarding the inter-individual distance network, no individual had high power in terms of eigenvector centrality.

\section{Discussion}

In the present study, we were able to characterize aspects of individual spatial positioning within a group of feral horses as a test case using a drone. We found a sex difference in spatial positioning in which a male was located toward the periphery of the group more frequently than females, at least during the 
breeding and birth season (our observation period). Distribution of inter-individual distances showed a regularity in that it fit with a gamma distribution. Additionally, spatial distributions were not random within the area, as indicated by the q-value that tended to be less than 0.5 . Nearest neighbors were located toward the sides of horses more often than toward the rear. Finally, grooming frequency and the spatial cohesion did not correlate with each other.

Our finding indicating that the distance from the central point to an individual did not differ among females contradicts a previous study which showed that subordinate horses were more likely to be located in the periphery of a group comprising a gelding and several females (Ingólfsdóttir and Sigurjónsdóttir 2008). The richness of food resources in our study site might have resulted in less competition among individuals. Center/periphery positioning is thought to affect foraging efficiency less when is food is depleted more slowly, which was the case for grass and herbs that covered this field site (Morrell and Romey 2008; Hirsch 2007). This could also explain the low frequency of aggressive behavior during our observation period. The tendency for the male to be located at the periphery could be related to a seasonal behavioral pattern. Thus, the male might have been attempting to defend females in the group from other males by staying in the periphery. This possibility is supported by previous research demonstrating that females choose males that protect them from harassment (Rubenstein 1994; Linklater et al. 1999). Notably, more than 20 other groups, including bachelor groups, were located in the same field (Ringhofer et al. 2017). To better understand the social and ecological factors influencing the positioning of horses, future studies collecting location data in non-breeding seasons will be necessary.

Our result regarding the distance to the nearest neighbor suggests that horses have a repulsion area with a 3-BL radius. The distribution of distances to the nearest neighbor did not follow a Weibull distribution. According to a Weibull distribution, which reflects a random distribution of points (Fortin et al. 2002), the distance between two points can be as close as possible to 0 . Thus, a repulsion area creates a non-Weibull (non-random) distribution. The result of the q-value analysis also supports the idea that individual horses were not located at random positions. The low frequency of q-values greater than 0.6 further suggests the possibility that individuals are attracted to each other within groups of horses. Thus, our results indicate that horses might have both areas of repulsion and attraction, similar to findings in flocks of birds and schools of fish, although the horses might also sometimes follow a random 
distribution as indicated by the $\sim 30 \%$ of cases in which the q-value was around 0.5 . However, we must keep in mind that our result is not direct evidence of repulsion and attraction areas, as we relied on photographic data of stationary positions, rather than measuring movements. Further studies with movement or velocity data are thus warranted.

The nearest neighbors of the horses were located on the sides more frequently than to the rear. Horses are able to see 350 degrees around themselves, but they have a blind spot directly behind them (Rees 1993). Therefore, horses may express fear of animals or objects grouping to their rear, which renders them invisible. Our results regarding the orientation of the nearest neighbors support this hypothesis. The average and minimum values of the inner product for the rear were higher than those for the front. This result indicates that individuals located behind others did not completely reverse their orientations to the individuals in front of them. This may be because horses tend to avoid putting animals in their blind spot or because they try to avoid being in the blind spots of other animals. Anisotropic positioning of the nearest neighbor has been reported in starlings and surf scoters (Ballerini et al. 2008; Lukeman et al. 2010; Pettit et al. 2013). But a direct comparison of our findings with horses and the previous study with starlings should be approached with caution because horses live in 2D space and starlings live in 3D space. On the other hand, a comparison between horses and surf scoters (Lukeman et al. 2010) would be more feasible because scoters in the cited study lived in 2D space (floated on the sea surface). Thus both horses and scoters lived in 2D space and can more easily be compared. Although our study had a similar research question to that by Lukeman et al. (2010), our results were somewhat different. In particular, the nearest neighbors of scoters were usually located to the front or rear, and most were within $2 \mathrm{BL}$. Preference for positioning with respect to the nearest neighbor seems to depend on the species, as well as on factors such as environment, the number of individuals in a group, and density of individuals.

Kimura (1998) reported that a partner with highest grooming frequency differed from the most frequent nearest neighbor in free-ranging horses. Our results from social network analysis showed mixed trends. The social networks measured by grooming frequency and proximity had a positive correlation, while the social network measured by distance (i.e., used as a quantitative measure) did not correlate with grooming networks. Individuals with high eigenvector centrality were different in each of 
the three networks. Grooming, proximity, and inter-individual distance might to some extent indicate different and independent social relationships between individuals. Observing one type of social interaction is clearly insufficient for describing horse society, and different types of indices can illustrate different aspects of horse society.

One limitation of our study is the short observation period. However, some factors favor short-term data acquisition for investigating social relationships in horses. First, addition of individuals due to the birth of new foals changes the positioning of individuals within a group. In the present study, we excluded the foals that were dependent on their mothers from the analysis (see Supplementary Material for the inclusion of foals in the analysis). However, horse foals grow rapidly. Thus, these foals must be included in the analysis at some point when their spatial positioning becomes independent. Second, immigration/emigration of individuals between groups is common (Linklater and Cameron 2009; see Ringhofer et al. 2017 for the data from the present study site), and the addition/loss of individuals will also affect spatial positioning within a group. Along with the passage of time after the addition of individuals, the relationships between individuals within a group can change. By the time the relationships have stabilized again, another immigration/emigration might occur. Therefore, the relationships of individual horses in our study area are constantly changing. Even if we collect long-term data, we would need to segment the data into short-term periods based on these reasons. With that said, long-term data on multiple groups of horses are necessary to verify our results, and we plan to conduct further research on related topics in the future.

In terms of using a drone, the advantages include higher accuracy and more objective data than can be recorded with human eyes at head height. Our method will be applicable to studies with many other species such as cows, elephants, and other ungulates that have been already monitored by the drone technology (Vermeulen et al. 2013; Chrétien et al. 2016; Goolsby et al. 2016; Torney et al. 2016; Jung 2017). At the same time however, the use of drones has several environmental limitations. First, flying a drone is impossible if the wind is too strong (approximately $>10 \mathrm{~m} / \mathrm{s}$ for the drone we used in our study) (DJI 2015). In fact, we experienced a situation in which our drone became uncontrollable and flew away in a sudden strong wind. Second, drones are best when applied to diurnal animals living in open areas. Recording forest-living animals via drone is difficult and hindered by trees. In addition, using drones to 
390

record nocturnal animals is still difficult, although using an infrared camera is a potential option. Third,

391 the battery-life of quadcopter-type drones (like the one used in our study) is only about 15 min, which is very short. Fixed-wing drones have a longer battery-life, but they are not capable of hovering, and thus recording animals that stay put in a given area is impossible with fixed-wing drones. Quadcopter drones are capable hovering but their short battery-life is certainly a limitation. Therefore, we have to take into consideration the short-battery life when we use drones for scientific studies. Indeed, this limitation was one of the factors that forced us to limit our analysis to analyze stationary situations. Fourth, quadcopter drones also make noise, thus it is not suitable for nervous animals that might react to the noise. Using drones thus has advantages and disadvantages, and our study has provided an example of what can be achieved when using them in animal studies. Our study has thus opened up new possibilities for studying animal behavior in the wild. 
Figure 1. Serra D’Arga and horses.

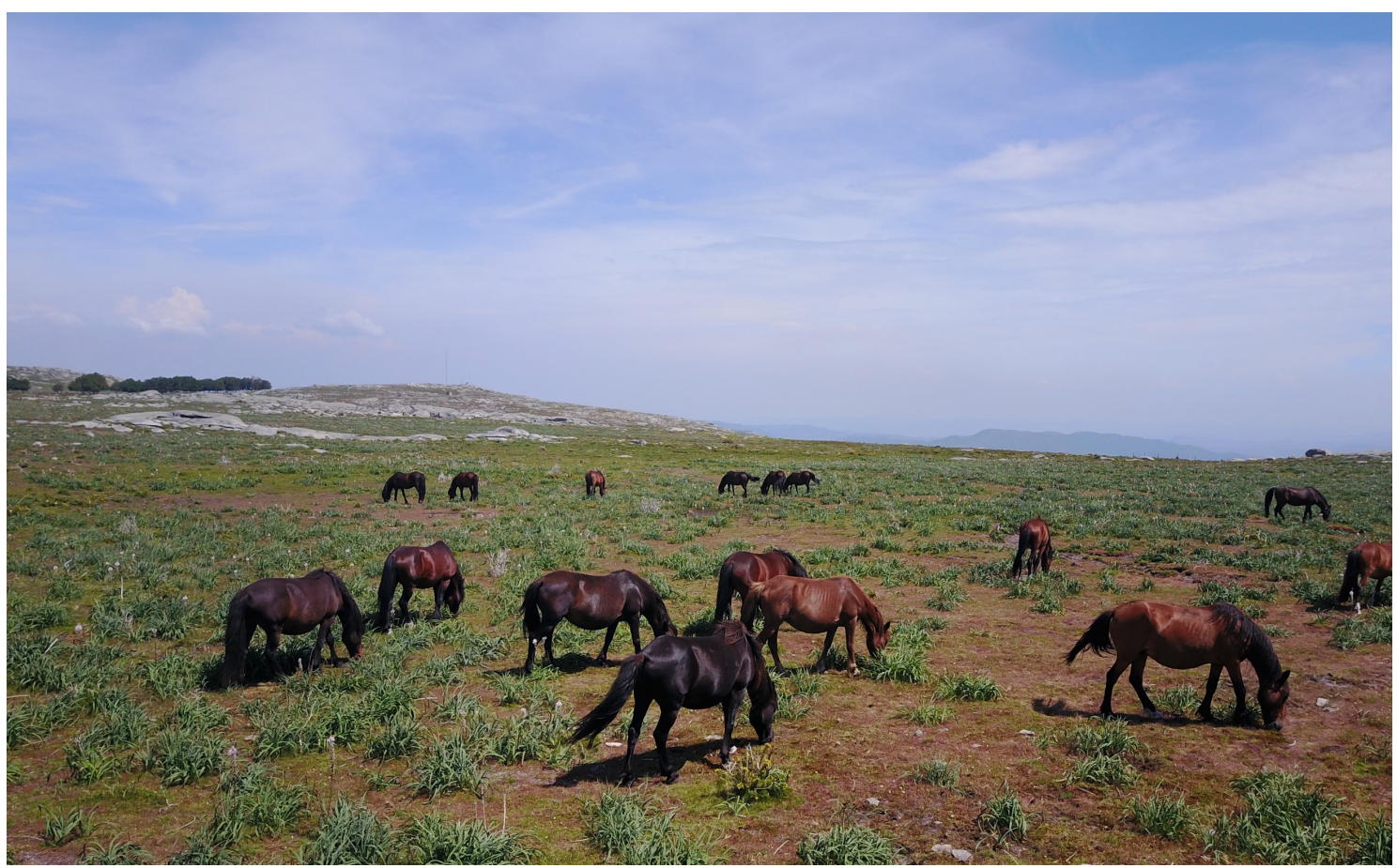

404

Figure 2. Drone picture of the Kyoto group.

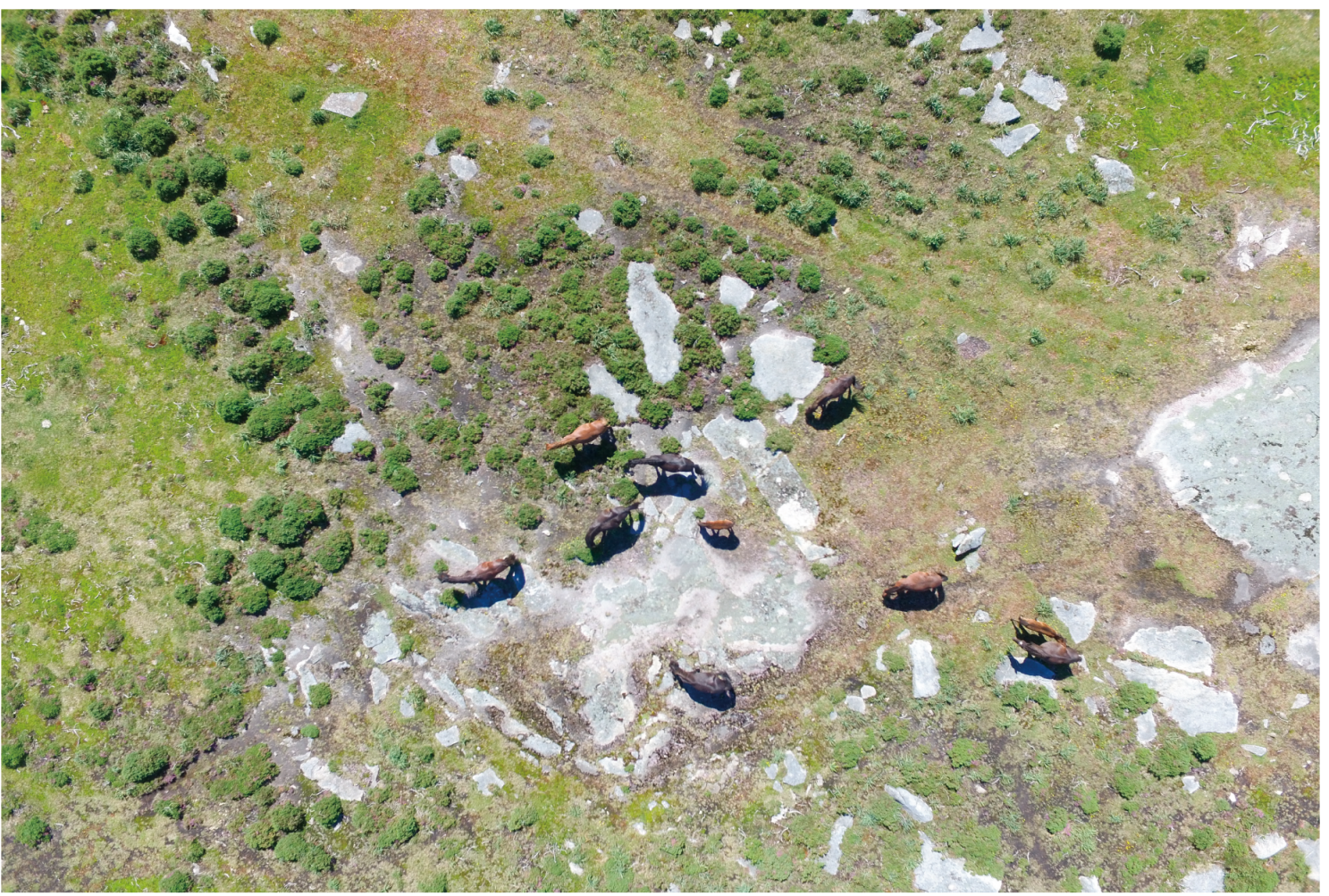



only male in this group.

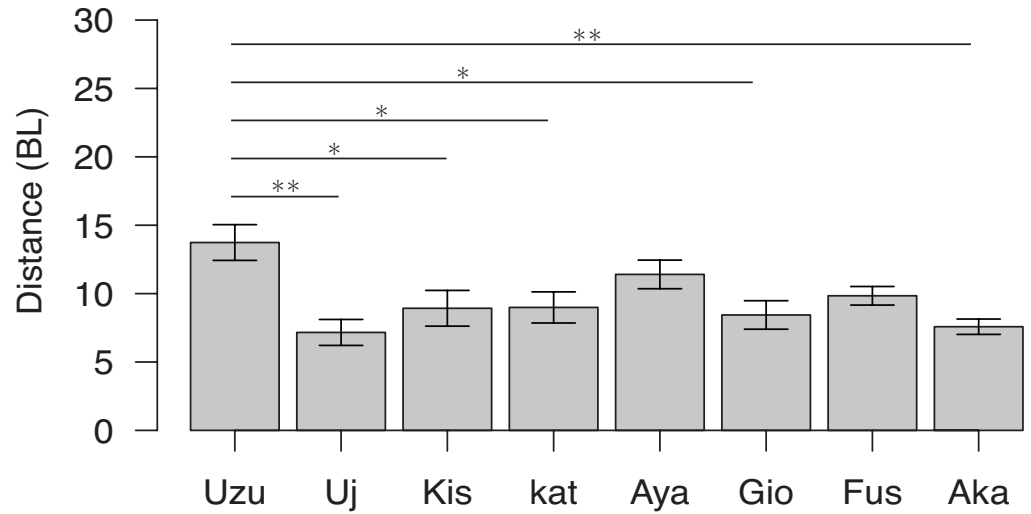

409

410 Figure 4. Histograms of inter-individuals distance. Bin size is 1 body length (BL). (A) Histogram of the 411 distances between all pairs. The peak of this histogram was around 4 BL. (B) Histogram of the distance to 412 nearest neighbor. The peak of this histogram was $3 \mathrm{BL}$.
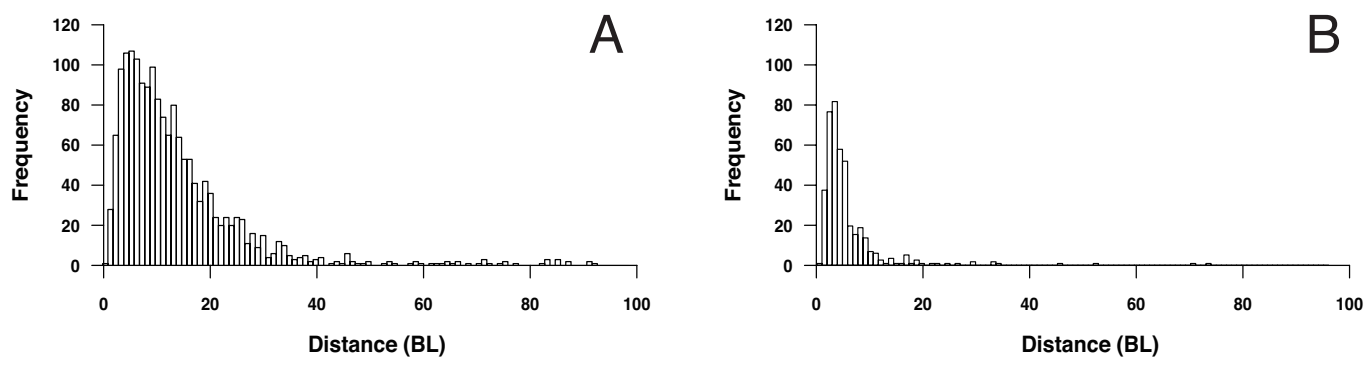

Figure 5. q-value histogram. 


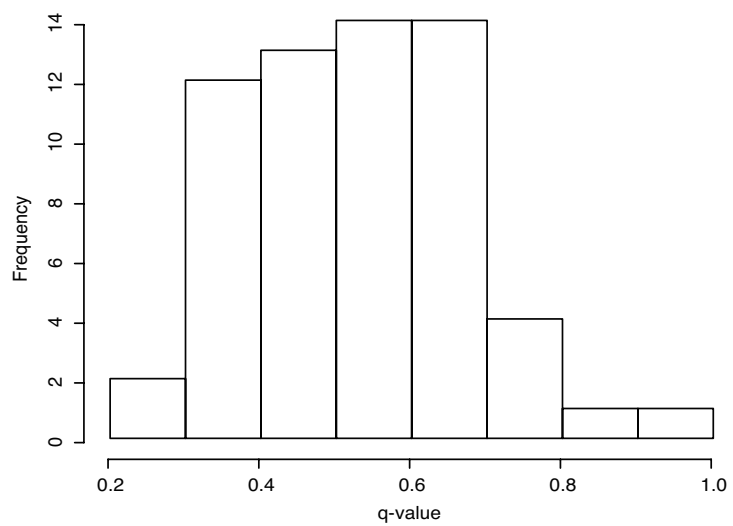

420 Figure 6. Density plot of nearest neighbor positions within 5 body lengths (BL).

421 We drew the density plot of the nearest neighbor based on pooled data from all individuals with the $\mathrm{R}$ 422 package "spatstat" (Baddely and Turner, 2007), with bin sizes of $\Delta x=\Delta y=0.5$ BL, and smoothening with a Gaussian Blur $=0.4$. If the nearest neighbors were located more than $10 \mathrm{BL}$ away, these cases were excluded from analysis because $91 \%$ of the data regarding the distance to the nearest neighbor fell within $10 \mathrm{BL}$.

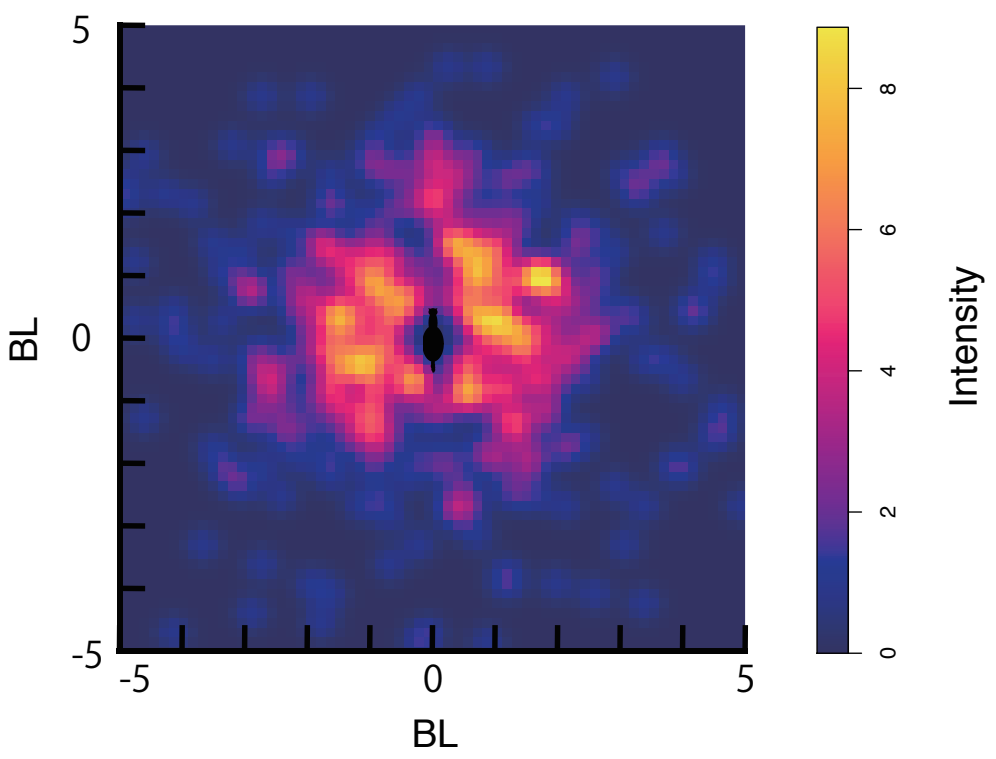

Figure 7. Horse social networks (a: distance, b: grooming and c: proximity). 


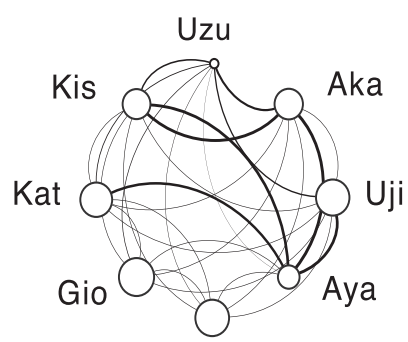

Fus

a. Distance

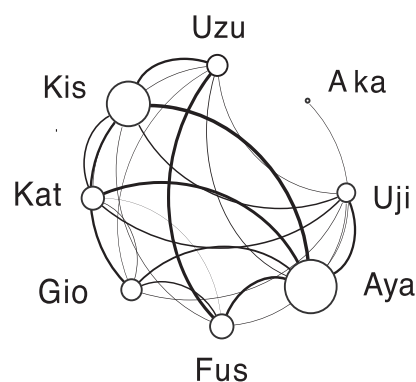

b. Grooming

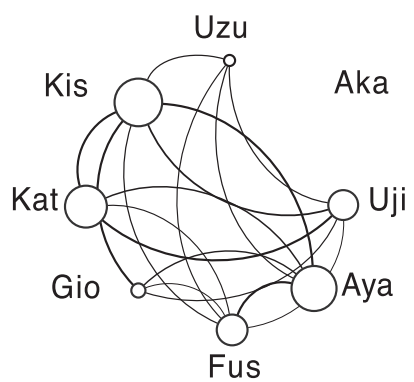

c. Proximity 
430

431 Supplemental Figure 1

432 Distribution of distances between foals and other individuals (Orange) compared with distances between

433 all individuals including foals (white), and distances between foals and their mothers (Red). The result

434 shows that the distances between foals and other individuals tended to be shorter than those between all

435 individuals. Additionally, the distribution of peak distances between foals and their mothers was shifted

436 to left compared with other distributions.

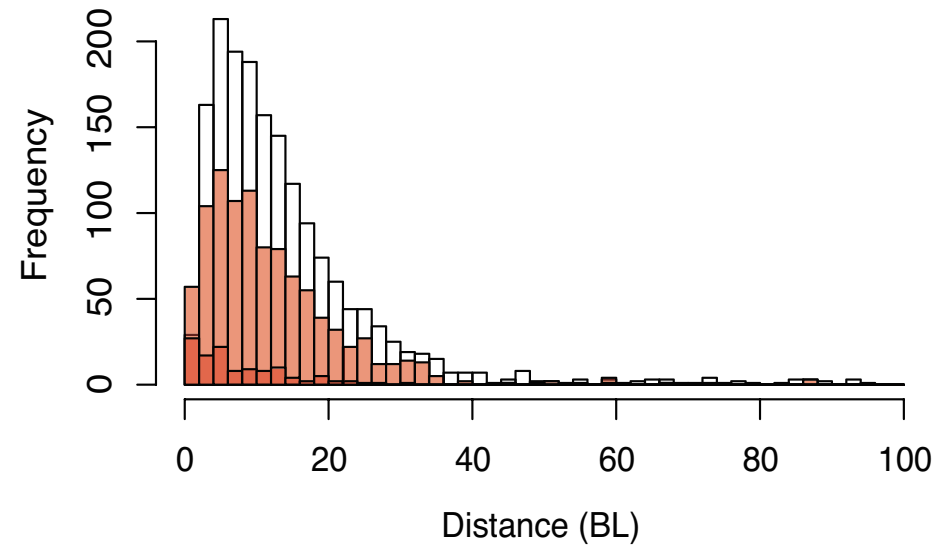




\section{References}

Altmann, J. (1974). Observational study of behavior: sampling methods. Behaviour, 49(3), 227-266.

Andrieu J, Henry S, Hausberger M, Thierry B (2016) Informed horses are influential in group movements, but they may avoid leading. Anim Cogn 19:451-458. doi: 10.1007/s10071-015-0945-2

Ballerini M, Cabibbo N, Candelier R, et al (2008) Interaction ruling animal collective behavior depends on topological rather than metric distance: Evidence from a field study. Pnas 105:1232-1237. doi: $10.1073 /$ pnas. 0711437105

Barak Y, Yom-Tov Y (1989) The advantage of group hunting in Kuhl's bat Pipistrellus kuhlii (Microchiroptera). J Zool London 219:670-675. doi: 10.1111/j.1469-7998.1989.tb02608.x

Berger J (1977) Organizational systems and dominance in feral horses in the Grand Canyon. Behav Ecol Sociobiol 2:131-146. doi: 10.1007/BF00361898

Bourjade M, Thierry B, Hausberger M, Petit O (2015) Is leadership a reliable concept in animals? An empirical study in the horse. PLoS One 10:1-14. doi: 10.1371/journal.pone.0126344

Borgatti, S. P., Everett, M. G., \& Freeman, L. C., 2002. Ucinet for Windows: Software for social network analysis.

Briard L, Dorn C, Petit O (2015) Personality and Affinities Play a Key Role in the Organisation of Collective Movements in a Group of Domestic Horses. Ethology 121:888-902. doi: 10.1111/eth. 12402

Cairns SJ, Schwager SJ (1987) A comparison of association indices. Anim Behav 35:1454-1469. doi: $10.1016 / \mathrm{S} 0003-3472(87) 80018-0$

Cameron EZ, Setsaas TH, Linklater WL (2009) Social bonds between unrelated females increase reproductive success in feral horses. Proc Natl Acad Sci U S A 106:13850-3. doi: 10.1073/pnas.0900639106

Chrétien L-P, Théau J, Ménard P (2016) Visible and thermal infrared remote sensing for the detection of white-tailed deer using an unmanned aerial system. Wildl Soc Bull 40:181-191. doi: $10.1002 / \mathrm{wsb} .629$

Couzin ID, Krause J, James R, et al (2002) Collective memory and spatial sorting in animal groups. J Theor Biol 218:1-11. doi: 10.1006/yjtbi.3065

Crowell-Davis SL, Houpt KA, Carini CM (1986) Mutual grooming and nearest-neighbor relationships among foals of Equus caballus. Appl Anim Behav Sci 15:113-123. doi: 10.1016/0168$1591(86) 90057-2$ 
Czaja JA, Ph D, Bielert C, Dj P (1975) Female Rhesus Sexual Behavior and Distance to a Male Partner : Relation to Stage of the Menstrual Cycle. 4:

Farine DR, Strandburg-Peshkin A, Couzin ID, et al (2017) Individual variation in local interaction rules can explain emergent patterns of spatial organization in wild baboons. Proc R Soc B Biol Sci 284:20162243. doi: $10.1098 / \mathrm{rspb} .2016 .2243$

Fortin M-J, Dale MRT, Hoef J (2002) Spatial analysis in ecology. Encycl Environmetrics 4:2051-2058

Furuichi T (1983) Interindividual Distance and Influence of Dominance on Feeding in a Natural Japanese Macaque Troop. 24:

Goolsby JA, Jung J, Landivar J, et al (2016) Evaluation of Unmanned Aerial Vehicles ( UAVs ) for detection of cattle in the Cattle Fever Tick Permanent Quarantine Zone. 24-27

Hamilton WD (1971) Geometry for the selfish herd. J Theor Biol 31:295-311. doi: 10.1016/00225193(71)90189-5

Harcourt AH (1978) Strategies of emigration and transfer by primates, with particular reference to gorillas. Z. Tierpsychol. 48:401-420

Heitor F, Oom M (2006) Social relationships in a herd of Sorraia horses Part II . Factors affecting affiliative relationships and sexual behaviours. 73:231-239. doi: 10.1016/j.beproc.2006.05.005

Heitor F, Oom M do M, Vicente L (2006) Social relationships in a herd of Sorraia horses. Part I. Correlates of social dominance and contexts of aggression. Behav Processes 73:170-177. doi: 10.1016/j.beproc.2006.05.004

Heitor F, Vicente L (2008) Maternal care and foal social relationships in a herd of Sorraia horses: Influence of maternal rank and experience. Appl Anim Behav Sci 113:189-205. doi: 10.1016/j.applanim.2007.11.005

Herskin J, Steffensen JF (1998) Energy savings in sea bass swimming in a school : measurements of tail beat frequency and oxygen consumption at di ff erent swimming speeds. J Fish Biol 53:366-376. doi: 10.1111/j.1095-8649.1998.tb00986.x

Hirsch, B. T. (2007). Costs and benefits of within-group spatial position: a feeding competition model. The Quarterly review of biology, 82(1), 9-27.

Hirsch BT (2011) Within-group spatial position in ring-tailed coatis: Balancing predation, feeding competition, and social competition. Behav Ecol Sociobiol 65:391-399. doi: 10.1007/s00265-010$1056-3$ 
Ingólfsdóttir HB, Sigurjónsdóttir H (2008) The benefits of high rank in the wintertime-A study of the Icelandic horse. Appl Anim Behav Sci 114:485-491. doi: 10.1016/j.applanim.2008.04.014 Janson CH (1990) Ecological Consequences of Individual spatial choices in foraging groups of brown capuchin monkeys, Cebus apella. Anim Behav 40:922-934. doi: 10.1016/S0003-3472(05)80994-7

Jung S (2017) Strategic Cattle Roundup using Multiple Quadrotor UAVs

Kano T (1982) The social group of pygmy chimpanzees (Pan paniscus) of Wamba. Primates 23:171-188. doi: $10.1007 / \mathrm{BF} 02381159$

Katz Y, Tunstrøm K, Ioannou CC, et al (2011) Inferring the structure and dynamics of interactions in schooling fish. doi: 10.1073/pnas.1107583108/-

/DCSupplemental.www.pnas.org/cgi/doi/10.1073/pnas.1107583108

Keiper RR (1988) Social interactions of the Przewalski horse (Equus przewalskii Poliakov, 1881) herd at the Munich Zoo. Appl Anim Behav Sci 21:89-97. doi: 10.1016/0168-1591(88)90102-5

Kimura R (1998) Mutual grooming and preferred associate relationships in a band of free-ranging horses. Appl Anim Behav Sci 59:265-276. doi: 10.1016/S0168-1591(97)00129-9

King AJ, Sueur C, Huchard E, Cowlishaw G (2011) A rule-of-thumb based on social affiliation explains collective movements in desert baboons. Anim Behav 82:1337-1345. doi: 10.1016/j.anbehav.2011.09.017

Klimov V V. (1988) Spatial-ethological organization of the herd of Przewalski horses (Equus przewalskii) in Askania-Nova. Appl Anim Behav Sci 21:99-115. doi: 10.1016/01681591(88)90103-7

Krueger K, Flauger B, Farmer K, Hemelrijk C (2014) Movement initiation in groups of feral horses. Behav Processes 103:91-101. doi: 10.1016/j.beproc.2013.10.007

Linklater W, Cameron E, Minot E, Stafford K (1999) Stallion harassment and the mating system of horses. Anim Behav 58:295-306. doi: 10.1006/anbe.1999.1155

Linklater WL, Cameron EZ (2009) Social dispersal but with philopatry reveals incest avoidance in a polygynous ungulate. Anim Behav 77:1085-1093. doi: 10.1016/j.anbehav.2009.01.017

Lukeman R, Li Y, Edelstein-keshet L (2010) Inferring individual rules from collective behavior. Pnas 107:12576-12580. doi: 10.1073/pnas.1001763107//DCSupplemental.www.pnas.org/cgi/doi/10.1073/pnas.1001763107

Morrell LJ, Romey WL (2008) Optimal individual positions within animal groups. Behav Ecol 19:909919. doi: 10.1093/beheco/arn050 
Nishida T (1968) The social group of wild chimpanzees in the Mahali Mountains. Primates 9:167-224. doi: 10.1007/BF01730971

Ozogány K, Vicsek T (2014) Modeling the Emergence of Modular Leadership Hierarchy During the Collective Motion of Herds Made of Harems. J Stat Phys 158:628-646. doi: 10.1007/s10955-014$1131-7$

Pettit, B., Perna, A., Biro, D., \& Sumpter, D. J. (2013). Interaction rules underlying group decisions in homing pigeons. Journal of The Royal Society Interface, 10(89), 20130529.Pollard JH (1971) On Distance Estimators of Density in Randomly Distributed Forests. Biometrics 27:991-1002

R Core Team (2016). R: A language and environment for statistical computing. R Foundation for Statistical Computing, Vienna, Austria. URL https://www.R-project.org/.

Rhine RJ, Bioland P, Lodwick L (1985) Progressions of adult male chacma baboons (Papio ursinus) in the Moremi Wildlife Reserve. Int J Primatol 6:115-122. doi: 10.1007/BF02693649

Ringhofer M, Inoue S, Mendonça RS, et al (2017) Comparison of the social systems of primates and feral horses: data from a newly established horse research site on Serra D'Arga, northern Portugal. Primates 58:479-484. doi: 10.1007/s10329-017-0614-y

Rosenthal SB, Twomey CR, Hartnett AT, et al (2015) Revealing the hidden networks of interaction in mobile animal groups allows prediction of complex behavioral contagion. Proc Natl Acad Sci 112:4690-4695. doi: 10.1073/pnas. 1420068112

Rubenstein, D. I., 1994. The ecology of female social behavior in horses, zebras, and asses. Animal societies: individuals, interactions, and organization, 13-28

Ryder TB, Horton BM, van den Tillaart M, et al (2012) Proximity data-loggers increase the quantity and quality of social network data. Biol Lett 8:917-20. doi: 10.1098/rsbl.2012.0536

Skellam, J. G., 1952. Studies in statistical ecology: I. Spatial Pattern. Biometrika, 39(3/4), 346-362. doi: $10.2307 / 2334030$

Strandburg-Peshkin A, Farine DR, Couzin ID, Crofoot MC (2015) Shared decision-making drives collective movement in wild baboons. Science (80- ) 348:1358-1361. doi: 10.1126/science.aaa5099

Strandburg-Peshkin A, Twomey CR, Bode NWF, et al (2013) Visual sensory networks and effective information transfer in animal groups. Curr Biol 23:R709-R711. doi: 10.1016/j.cub.2013.07.059

Sueur C, Deneubourg JL (2011) Self-Organization in Primates: Understanding the Rules Underlying Collective Movements. Int J Primatol 32:1413-1432. doi: 10.1007/s10764-011-9520-0 
559 Teichroeb JA, White MMJ, Chapman CA (2015) Vervet (Chlorocebus pygerythrus) Intragroup Spatial Positioning: Dominants Trade-Off Predation Risk for Increased Food Acquisition. Int J Primatol 36:154-176. doi: 10.1007/s10764-015-9818-4

Tokuyama N, Furuichi T (2017) Leadership of old females in collective departures in wild bonobos (Pan paniscus) at Wamba. Behav Ecol Sociobiol 71:. doi: 10.1007/s00265-017-2277-5

Torney CJ, Dobson AP, Borner F, et al (2016) Assessing rotation-invariant feature classification for automated wildebeest population counts. PLoS One 11:1-10. doi: 10.1371/journal.pone.0156342

Van Noordwijk, M. A., \& Van Schaik, C. P., 1987. Competition among female long-tailed macaques, Macaca fascicularis. Animal Behaviour, 35(2), 577-589. doi: 10.1016/S0003-3472(87)80284-1

Vermeulen, C., Lejeune, P., Lisein, J., Sawadogo, P., \& Bouché, P., 2013. Unmanned aerial survey of elephants. PloS one, 8(2), e54700. doi:10.1371/journal.pone.0054700

Wanng, G.H. (1983) Horse Behaviour: The Behavioral Traits and Adaptations of Domesric and Wild Horses, including Ponies,. Noyes Publications, New Jersey. pp 175-215.

Weeks JW, Crowell-Davis SL, Caudle AB, Heusner GL (2000) Aggression and social spacing in light horse (Equus caballus) mares and foals. Appl Anim Behav Sci 68:319-337. doi: 10.1016/S01681591(99)00126-4

Weimerskirch H, Martin J, Clerquin Y, et al (2001) Energy saving in flight formation. Nature 413:697-8. doi: $10.1038 / 35099670$ 